OUT-4102-85

$\mathrm{MZ}-\mathrm{TH} / 00-01$

hep-th/0001202

28 January 2000

\title{
Towards cohomology of renormalization: bigrading the combinatorial Hopf algebra of rooted trees
}

\author{
D. J. Broadhurst ${ }^{1)}$ and D. Kreimer ${ }^{2)}$
}

\begin{abstract}
The renormalization of quantum field theory twists the antipode of a noncocommutative Hopf algebra of rooted trees, decorated by an infinite set of primitive divergences. The Hopf algebra of undecorated rooted trees, $\mathcal{H}_{R}$, generated by a single primitive divergence, solves a universal problem in Hochschild cohomology. It has two nontrivial closed Hopf subalgebras: the cocommutative subalgebra $\mathcal{H}_{\text {ladder }}$ of pure ladder diagrams and the Connes-Moscovici noncocommutative subalgebra $\mathcal{H}_{\mathrm{CM}}$ of noncommutative geometry. These three Hopf algebras admit a bigrading by $n$, the number of nodes, and an index $k$ that specifies the degree of primitivity. In each case, we use iterations of the relevant coproduct to compute the dimensions of subspaces with modest values of $n$ and $k$ and infer a simple generating procedure for the remainder. The results for $\mathcal{H}_{\text {ladder }}$ are familiar from the theory of partitions, while those for $\mathcal{H}_{\mathrm{CM}}$ involve novel transforms of partitions. Most beautiful is the bigrading of $\mathcal{H}_{R}$, the largest of the three. Thanks to Sloane's superseeker, we discovered that it saturates all possible inequalities. We prove this by using the universal Hochschild-closed one-cocycle $B_{+}$, which plugs one set of divergences into another, and by generalizing the concept of natural growth beyond that entailed by the Connes-Moscovici case. We emphasize the yet greater challenge of handling the infinite set of decorations of realistic quantum field theory.
\end{abstract}

1) D.Broadhurst@open.ac.uk; http://physics.open.ac.uk/ dbroadhu Physics Dept, Open University, Milton Keynes MK7 6AA, UK

2) Dirk.Kreimer@uni-mainz.de; http://dipmza.physik.uni-mainz.de/kreimer Heisenberg Fellow, Physics Dept, Univ. Mainz, 55099 Mainz, Germany 


\section{Introduction}

In this paper we bigrade the Hopf algebra of undecorated rooted trees, and both of its closed Hopf subalgebras, taking account of Hochschild cohomology.

In [1]-[9] we have exposed the connection between renormalization and Hopf algebra. The joblist of renormalization specifies a noncocommutative coproduct, $\Delta$. On the left are products of divergent subdiagrams; on the right these shrink to points. An antipode, $S$, upgrades this bialgebra to a Hopf algebra, by specifying the procedure of subtracting subdivergences. If this antipode is twisted, by taking only the poles of the Laurent series in $\varepsilon$, for dimensionally regularized diagrams in $d:=4-2 \varepsilon$ spacetime dimensions, the final subtraction delivers a finite renormalized Green function, in the limit $d \rightarrow 4$, corresponding to the minimal subtraction scheme. Different twists correspond to different renormalization schemes [1].

The general problem of perturbative quantum field theory involves the Hopf algebra of decorated rooted trees. These decorations represent primitive divergences, coming from diagrams with no subdivergences. Restriction to the Hopf algebra $\mathcal{H}_{R}$ of undecorated rooted trees, generated by a single primitive divergence, reveals a remarkable feature. This apparently small problem in quantum field theory has a mathematical structure larger than a very general problem in noncommutative geometry, investigated by Alain Connes and Henri Moscovici [10], who showed that the composition of diffeomorphisms can be described algebraically, and hence extended to noncommutative manifolds, by making use of an appropriate Hopf algebra $\mathcal{H}_{\mathrm{CM}}$. In [2] it was shown that the Hopf algebra $\mathcal{H}_{\mathrm{CM}}$ of [10] is, in the one-dimensional case, the unique noncocommutative Hopf subalgebra of $\mathcal{H}_{R}$, corresponding to adding Feynman diagrams [4] with weights determined by natural growth. The only other closed Hopf subalgebra is the cocommutative Hopf algebra $\mathcal{H}_{\text {ladder }}$ of rooted trees whose nodes have fertility less than 2 , corresponding to the ladder (or rainbow) diagrams of [1]]-[14].

Suppose we are given an $n$-loop Feynman diagram that represents an $n$-node tree in $\mathcal{H}_{R}$. It involves $n$ ultraviolet-divergent integrations, and hence one may expect that it delivers, in dimensional regularization, a pole of $n$-th order. But then, combinations of diagrams corresponding to sums of products of rooted trees can provide cancellations of poles, and may hence eliminate leading pole terms. A prominent example of such a mechanism occurs in the calculation of an anomalous dimension, $\gamma=d \log Z / d \log \mu$, which detects only single-pole terms, after minimal subtraction of subdivergences. All higher order poles are determined by the requirement that they cancel when one takes the derivative of the logarithm of the renormalization factor $Z$ w.r.t. to the renormalization scale $\mu$. Every practitioner of multiloop quantum chromodynamics is vividly aware of the bigrading of her/his work, by loop number and degree of singularity. The slightest error in handling either the combinatorics or the integrations usually - and mercifully reveals itself by a failure to get the uniquely finite answer that is ensured by the locality of counterterms. Thus there is deep - and largely uncharted - structure in the relations between Laurent expansions of products of Feynman diagrams, corresponding to forests of rooted trees. 
In this work we make preparation for a cohomological approach to renormalization, by identifying and analyzing a combinatoric bigrading of linear combinations of undecorated rooted forests.

In sect. 2, we define this bigrading, in terms of the number of nodes $n$ and an index $k$ that classifies subspaces according to their projection into an augmentation ideal, analyzed by $k$-fold iterations of the coproduct $\Delta$. We wish to learn the dimension, $H_{n, k}$, of the subspace with weight $n$ and index $k$. In sect. 3 we find that this problem has a very simple solution in the cocommutative subalgebra $\mathcal{H}_{\text {ladder }}$, where the dimension is the number of ways of partitioning $n$ into $k$ positive integers, given in Table 1 . In sect. 4 we find that the corresponding problem in the noncocommutative subalgebra $\mathcal{H}_{\mathrm{CM}}$ has the subtler solution of Table 2, which we find to be related to Table 1 by a remarkable transform, which preserves the sums of rows. In sect. 5 we team Neil Sloane's superseeker [15] with Tony Hearn's Reduce [16] and find

$$
H(x, y):=\sum_{n, k} H_{n, k} x^{n} y^{k}=\frac{R(x)}{(1-y) R(x)+x y}
$$

for the generating function of the bigrading of $\mathcal{H}_{R}$, with results in Table 3 obtained from [17]

$$
R(x):=\sum_{n>0} r_{n} x^{n}=x \prod_{n>0}\left(1-x^{n}\right)^{-r_{n}}=x+x^{2}+2 x^{3}+4 x^{4}+9 x^{5}+20 x^{6}+\ldots
$$

which generates the number $r_{n}$ of rooted trees with $n$ nodes. Our discovery of the generating principle of Table 3 was triggered by superseeker analysis of merely the first 8 entries of its first column. After thorough study of the filtration in Table 4, we prove (1).

\section{The second grading}

The weight $n$ of a rooted tree $t$ is the number of its nodes. The weight of a forest $F=\prod_{j} t_{j}$ is the sum of the weights of the trees $t_{j}$ in the product. This is the first grading.

To define the index $k$ for the second grading, $k$-primitivity, we use $k$-fold iterations of the coproduct $\Delta$, defined by the highly nontrivial recursion [1]

$$
\Delta(t)=t \otimes e+\left(\mathrm{id} \otimes B_{+}\right) \circ \Delta \circ B_{-}(t)
$$

for a nonempty tree $t$. Here $e$ is the empty tree, evaluating to unity, id is the identity map, $B_{-}$removes the root of $t$, and $B_{+}$combines the trees of a product by appending them to a common root. The coproduct $\Delta$ is coassociative. Hence it has a unique iteration, which may be written in a variety of equivalent ways. Since $\Delta$ has only single trees on the right, the recursion

$$
\Delta^{k}=\left(\mathrm{id} \otimes \Delta^{k-1}\right) \circ \Delta
$$

is particularly convenient. For a forest $F=\prod_{j} t_{j}$ we have $\Delta^{k}(F)=\prod_{j} \Delta^{k}\left(t_{j}\right)$.

Let $X$ be a $\mathbb{Q}$-linear combination of monomials of trees, i.e. of forests. We say that $X$ is $k$-primitive if every term of $\Delta^{k}(X)$ has at least one empty tree $e$. Symbolically we may 
consider the composition of tensor products of the projection operator $P:=\mathrm{id}-E \circ \bar{e}$ with iterations of $\Delta$. P projects onto the augmentation ideal $\mathcal{H}_{c}=\left\{X \in \mathcal{H}_{R} \mid P(X)=X\right\}$, where $X=P(X)+E \circ \bar{e}(X)$. Here $\bar{e}$ is the counit, which annihilates everything except the empty tree, for which it gives $\bar{e}(e)=1$. The map from the rationals back to the algebra is simply $E(q)=q e$ for $q \in \mathbb{Q}$. Hence $P$ annihilates $e$ and leaves everything else unchanged. Let $U_{0}:=P$ and

$$
U_{k}:=(\underbrace{P \otimes \ldots \otimes P}_{k+1 \text { times }}) \circ \Delta^{k}=\left(P \otimes U_{k-1}\right) \circ \Delta
$$

for $k>0$. In using the recursive form, note should be taken that, in general, the projection makes $U_{k}\left(X_{1} X_{2}\right) \neq U_{k}\left(X_{1}\right) U_{k}\left(X_{2}\right)$ : one should store results for forests; not just for trees.

We have said that $X$ is $k$-primitive if $U_{k}(X)=0$. Then clearly $X$ is $(k+1)$-primitive, since $\Delta^{k+1}(X)$ has at least two empty trees $e$ in every term. We are interested in the number, $H_{n, k}:=D_{n, k}-D_{n, k-1}$, of weight- $n$ terms that are $k$-primitive but are not $(k-1)$ primitive, where $D_{n, k}$ is the dimension of the subspace with weight $n$ and index $k$. To compute $D_{n, k}$ for specific (and rather modest) values of $n, k$ one considers the most general linear combination $X$ of weight- $n$ terms, with unknown coefficients, and solves $U_{k}(X)=0$. The rank deficiency of this large system of linear equations is $D_{n, k}$. From this one subtracts the number $D_{n, k-1}$ of weight- $n$ terms that are $(k-1)$-primitive. By this means we obtained the first 7 rows of Tables 1 and 2, for the Hopf subalgebras $\mathcal{H}_{\text {ladder }}$ and $\mathcal{H}_{\mathrm{CM}}$, and inferred their generating principles. In the case of the full Hopf algebra $\mathcal{H}_{R}$, bigraded in Table 3 , data were much harder to obtain. Fortunately the generating principle is very distinctive.

\section{Bigrading the cocommutative subalgebra}

We first consider the cocommutative Hopf algebra $\mathcal{H}_{\text {ladder }}$ of rooted trees all of whose nodes have fertility less than 2, i.e. the Hopf algebra with linear basis $l_{n}=B_{+}^{n}(e), n \geq 0$. In this very simple case, the recursive definition (3) linearizes on the left, giving

$$
\Delta\left(l_{n}\right)=\sum_{k=0}^{n} l_{n-k} \otimes l_{k}
$$

for the unique $n$-node tree $l_{n} \in \mathcal{H}_{\text {ladder. }}$. Thanks to our recent work in [8] we have an explicit construction of the weight- $n$ 1-primitive $p_{n} \in \mathcal{H}_{\text {ladder. }}$. First we compute the antipodes. In the cocommutative case, these are simply

$$
S\left(l_{n}\right)=-\sum_{k=0}^{n-1} S\left(l_{n-k}\right) l_{k}
$$

with $l_{0}=e$ and $S(e)=e$. To construct the 1-primitives, we use the star product $S \star Y$, where $Y$ is the grading operator, giving $Y\left(l_{k}\right)=k l_{k}$. In general, a star product of operators is defined by $O_{1} \star O_{2}:=m \circ\left(O_{1} \otimes O_{2}\right) \circ \Delta$, where $m$ merely multiplies entries on the left and right of a tensor product. The ladder 1-primitives are given by

$$
p_{n}:=\frac{1}{n}[S \star Y]\left(l_{n}\right)=\sum_{k=0}^{n} \frac{k}{n} S\left(l_{n-k}\right) l_{k} .
$$


Clearly $p_{1}=l_{1}$ and $p_{2}=l_{2}-\frac{1}{2} l_{1}^{2}$ are 1 -primitive. It takes some time to show that

$$
\begin{aligned}
p_{8}= & l_{8}-l_{7} l_{1}-l_{6} l_{2}+l_{6} l_{1}^{2}-l_{5} l_{3}+2 l_{5} l_{2} l_{1}-l_{5} l_{1}^{3}-\frac{1}{2} l_{4}^{2}+2 l_{4} l_{3} l_{1}+l_{4} l_{2}^{2}-3 l_{4} l_{2} l_{1}^{2}+l_{4} l_{1}^{4} \\
& +l_{3}^{2} l_{2}-\frac{3}{2} l_{3}^{2} l_{1}^{2}-3 l_{3} l_{2}^{2} l_{1}+4 l_{3} l_{2} l_{1}^{3}-l_{3} l_{1}^{5}-\frac{1}{4} l_{2}^{4}+2 l_{2}^{3} l_{1}^{2}-\frac{5}{2} l_{2}^{2} l_{1}^{4}+l_{2} l_{1}^{6}-\frac{1}{8} l_{1}^{8}
\end{aligned}
$$

gives $\Delta\left(p_{8}\right)=p_{8} \otimes e+e \otimes p_{8}$. We were able to compute this primitive with ease, using recursion (7) in the star product (8). From [8] we know that $[S \star Y](t)$ delivers a combination of diagrams whose singularity is a single pole, as $d \rightarrow 4$, with a residue that determines the contribution of $t$ to the anomalous dimension. Moreover 1-primitives have only single poles. However the converse is not true in the full Hopf algebra: noncocommutativity implies that not every $[S \star Y](t)$ is 1-primitive. Here, in the cocommutative subalgebra, there is a single 1-primitive for each weight $n>0$. Hence $S \star Y$ delivers it.

From examples such as (9) we inferred the general result of (8). The 1-primitive $p_{n}$ contains all possible multiplicative partitions $\prod_{j} l_{j}^{n_{j}}$ with weight $n=\sum_{j} n_{j} j$. The coefficient of each partition is $(-1)^{k-1}(k-1) ! / \prod_{j} n_{j}$ ! where $k=\sum_{j} n_{j}$ is the number of integers into which $n$ has been partitioned. For example the partition $8=2+2+1+1+1+1$, with $k=6$, gives the coefficient $-5 ! / 2 ! 4 !=-5 / 2$ of $l_{2}^{2} l_{1}^{4}$ in (9). We have tested this Ansatz up to $n=20$, where $p_{20}$ contains 627 terms.

It is easy to understand the leading diagonal of Table $1: l_{1}^{n}$ is $n$-primitive, but not $(n-1)$-primitive. For $8>n>k>1$ we used Reduce to prove the results of Table 1 . The entry in the $n$-th row and $k$-th column is $\bar{H}_{n, k}=\bar{D}_{n, k}-\bar{D}_{n, k-1}$, where $\bar{D}_{n, k}$ is the number of undetermined coefficients when one solves $U_{k}(X)=0$, with $X$ taken as an unknown linear combination of forests $\prod_{j} l_{j}^{n_{j}}$ of weight $n=\sum_{j} n_{j} j$. Clearly the generating principle is extremely simple: $\bar{H}_{n, k}$ is the number of partitions of $n$ into $k$ positive integers. This simply reflects the fact that solving $U_{k}(X)=0$ determines all and only the coefficients of partitions with $\sum_{j} n_{j} \leq k$. Hence the $k$-th column of Table 1 is generated by

$$
\bar{H}_{k}(x):=\sum_{n} \bar{H}_{n, k} x^{n}=\prod_{j \leq k} \frac{x}{1-x^{j}}=\frac{x}{1-x^{k}} \bar{H}_{k-1}(x)
$$

which yields the recursion of the tabular entry A048789 of [15]:

$$
\bar{H}_{n, k}=\bar{H}_{n-k, k}+\bar{H}_{n-1, k-1}
$$

seeded by the empty tree, which gives $\bar{H}_{0,0}=1$. We particularly note that for all $j, k>0$

$$
\bar{H}_{j+k}(x)<\bar{H}_{j}(x) \bar{H}_{k}(x) .
$$

\section{Bigrading the Connes-Moscovici subalgebra}

To compute Table 2 we proceeded as above, now using the coproduct [2]

$$
\begin{aligned}
\Delta\left(\delta_{n}\right) & =\delta_{n} \otimes e+e \otimes \delta_{n}+R_{n-1} \\
R_{n} & =\left[\mathbf{X} \otimes e+e \otimes \mathbf{X}+\delta_{1} \otimes \mathbf{Y}, R_{n-1}\right]+\delta_{1} \otimes Y\left(\delta_{n}\right)
\end{aligned}
$$


with $R_{0}=0,\left[\mathbf{X}, \delta_{n}\right]=\delta_{n+1}$ increasing weight, and $\left[\mathbf{Y}, \delta_{n}\right]=Y\left(\delta_{n}\right)=n \delta_{n}$ measuring weight. This is the noncocommutative coproduct of Connes and Moscovici [10], shown in [2] to give the closed Hopf subalgebra of $\mathcal{H}_{R}$ that is realized by $\delta_{n}=N^{n-1}\left(l_{1}\right)$, where $N$ is the natural growth operator, which appends a single node in all possible ways. Thus $\delta_{1}=l_{1}$ and $\delta_{2}=l_{2}$, while $\delta_{3}=l_{3}+B_{+}\left(l_{1}^{2}\right)$ differs from the ladder-algebra element $l_{3}$. Natural growth implies that $\delta_{n}$ is a sum over all weight- $n$ trees in $\mathcal{H}_{R}$, with nonzero Connes-Moscovici weights that we specified in [4], using an efficient recursive procedure.

Computation of the first 7 rows of Table 2 took longer than for Table 1, because of the proliferation of product terms on the left of the noncocommutative coproduct. These scanty data presented us with a pretty puzzle, which the reader might like to try to solve, after covering up the rows of Table 2 with $n>7$. What is the generating procedure? Recall that the sum of the $n$-th row in Table 2 must agree with that in Table 1 , since each gives the total number of ways of partitioning the integer $n$. In Table 1 this is achieved with great simplicity: the $k$-th entry is the number of ways of partitioning $n$ into $k$ positive integers. In Table 2 it is achieved far more subtly, by the addition of only $1+\lfloor n / 2\rfloor$ terms, since $\widetilde{H}_{n, k}$ has support only for $2 k \geq n \geq k$.

Given merely data for $n \leq 7$, the most interesting feature is the second subleading diagonal $1,2,4,6 \ldots$ The leading diagonal is generated by $G_{0}=1 /(1-z)$, the first subleading diagonal by $G_{1}=1 /(1-z)^{2}$. The simplest Ansatz for the second is $G_{2}=G_{1} /\left(1-z^{2}\right)$, which requires $\widetilde{H}_{8,6}=9$. Then $\widetilde{H}_{8,5}=4$ is required, so that $1+\widetilde{H}_{8,5}+9+7+1=22$ is the number of ways of partitioning 8. A Reduce program, running for 24 hours, proved that indeed $\widetilde{H}_{8,5}=4$. Next, the requirement $\widetilde{H}_{9,6}=7$ comes from $2+\widetilde{H}_{9,6}+12+8+1=30$, for the partitions of 9 , taking $\widetilde{H}_{9,7}=12$ from the hypothesis $G_{2}=1 /(1-z)^{2}\left(1-z^{2}\right)$ for the second subleading diagonal. Then the third subleading diagonal is revealed as $1,2,4,7 \ldots$ which is nicely consistent with $G_{3}=G_{2} /\left(1-z^{3}\right)$. Finally, it is easy to check that the recurrence relation $G_{k}=G_{k-1} /\left(1-z^{k}\right)$ for the diagonals makes the rows sum to the correct partitions. Later we shall prove this result by considering the Connes-Moscovici restriction of the filtration of the bigrading of the full Hopf algebra.

In words, the transformation is simple to state: the subleading diagonals of Table 2 are the partial sums of the columns of Table 1. This leads to the subtle recurrence relation

$$
\widetilde{H}_{n, k}=\widetilde{H}_{k, 2 k-n}+\widetilde{H}_{n-2, k-1}
$$

for the bigrading of the Connes-Moscovici Hopf subalgebra. We particularly note that for $j, k>0$ and $j+k>2$

$$
\widetilde{H}_{j+k}(x)<\widetilde{H}_{j}(x) \widetilde{H}_{k}(x)
$$

while for $j=k=1$ we have the equality $\widetilde{H}_{2}(x)=\widetilde{H}_{1}(x) \widetilde{H}_{1}(x)=x^{2}(1+x)^{2}$.

\section{Bigrading the full Hopf algebra of rooted trees}

Given how long it took to compute the data that eventually led to the generating principle for the Connes-Moscovici subalgebra, one might be daunted by the task of inferring the 
bigrading of the full Hopf algebra of undecorated rooted trees. In fact, we discovered this first, by mere consideration of the first 8 entries in the first column of Table 3 . Thanks to [4] we had an extremely efficient Reduce implementation of the coproduct (3). The severity of the challenge of understanding the range and kernel of $U_{k}$, i.e. the difficulty of the computation of $\Delta^{k}$, increases drastically with $k$. At $k=1$ it was possible to solve $U_{1}(X):=(P \otimes P)(\Delta(X))=0$, for weights $n \leq 8$, using a few hours of CPUtime, notwithstanding the fact that at $n=8$ the number of products of trees is $r_{9}=286$.

The book-keeping was very simple, since the defining property of rooted trees is that every weight- $n$ forest $F=\prod_{j} t_{j}$ is uniquely labelled by the tree $B_{+}(F)$ with weight $n+1$. This clearly leads to the enumeration (2). More deeply, it shows that [2] $B_{+}$is Hochschild closed, and hence that the apparently simplistic quantum-field-theory task of handling a single primitive divergence solves a universal problem in Hochschild cohomology.

Submitting 1,1,1,2,3,8,16,41 to Neil Sloane's superseeker [15], we learnt that it is generated by the first 8 terms of

$$
H_{1}(x)=\frac{R(x)-x}{R(x)}=1-\prod_{n>0}\left(1-x^{n}\right)^{r_{n}} .
$$

At the time, Sloane had no idea that we were studying the bigrading of rooted trees and told us "it is pretty unlikely this is your sequence, but I thought I should pass this along just in case". In fact, his superseeker discovery unlocked our puzzle. We knew that

$$
\frac{R(x)}{x}=\sum_{k \geq 0} H_{k}(x)
$$

where $H_{0}(x):=1$ and $H_{k}(x):=\sum_{k} H_{n, k} x^{n}$ generates column $k$ of Table 3 . We then construed (17) as

$$
\frac{R(x)}{x}=\frac{1}{1-H_{1}(x)}=\sum_{k \geq 0}\left[H_{1}(x)\right]^{k} .
$$

Comparison with (18) then led to the conjecture $H_{k}(x)=\left[H_{1}(x)\right]^{k}$, requiring that

$$
H_{j+k}(x)=H_{j}(x) H_{k}(x) .
$$

To test this, we made intensive use of Reduce. At weight $n=9$ we computed the $3214 \times 719$ matrix of integer contributions to the 3214 terms in $\Delta(X)$ produced by $r_{10}=719$ weight-9 forests. The rank deficiency of the condition $U_{1}(X)=0$ was proven to be $D_{9,1}=98$, which is indeed the coefficient of $x^{9}$ in (17). We tested $H_{2}(x)=\left[H_{1}(x)\right]^{2}$ up to weight $n=8$, where $\Delta^{2}(X)$ has 3651 terms in 286 unknowns. Here $U_{2}(X)=0$ gave $D_{8,2}=41+58=99$, where 41 and 58 are indeed the coefficients of $x^{8}$ in (17) and its square. Finally, we tested $H_{3}(x)=H_{1}(x) H_{2}(x)$ up to weight $n=7$, where $\Delta^{3}(X)$ has 3168 terms in 115 unkowns, with $U_{3}(X)=0$ giving $D_{7,3}=16+26+27=69$, in agreement with the sum of the coefficients of $x^{7}$ in (17), its square and cube.

Hence we obtained compelling evidence for the bigrading (11) of the Hopf algebra of rooted trees, determined by the circumstance (20) that it saturates all inequalities. First we derive these general inequalities, for any commutative graded Hopf algebra. Then we prove that they are saturated in $\mathcal{H}_{R}$. 


\subsection{General inequalities}

Let $\mathcal{H}$ be a commutative graded Hopf algebra with unit $e$. Let deg be the grading, with $\operatorname{deg}(X) \in \mathbb{N}$ for all $X \in \mathcal{H}$ and $\operatorname{deg}(e)=0$. We assume that $\mathcal{H}$ is reduced to scalars by the counit $\bar{e}$.

Let $\mathcal{H}_{k}$ be the set of elements in the kernel of $U_{k}$ which are in the range of $U_{k-1}$, so that $U_{k}(X)=0$ and $U_{k-1}(X) \neq 0$, for $X \in \mathcal{H}_{k}$. Then we call $k$ the degree of primitivity of $X$, writing $\operatorname{deg}_{p}(X)=k$. We let $\mathcal{H}_{0}$ be the set of elements in the kernel of $P=U_{0}$, i.e. the scalars. The augmentation ideal fulfills $\mathcal{H}_{c}=\mathcal{H} / \mathcal{H}_{0}=\sum_{k=1}^{\infty} \mathcal{H}_{k}$.

To show that $\operatorname{deg}_{p}(X) \leq \operatorname{deg}(X)$, suppose that

$$
\Delta^{\operatorname{deg}(X)-1}(X)=\sum_{i} X_{i}^{(1)} \otimes \ldots \otimes X_{i}^{(\operatorname{deg}(\mathrm{X}))}
$$

has nonscalar entries in $\mathcal{H}_{c}^{\otimes \operatorname{deg}(X)}$. Then they are all formed from 1-primitives, in $\mathcal{H}_{1}$, since the coproduct is homogenous in $\operatorname{deg}$ and any element $X$ with $\operatorname{deg}(X)=1$ also has $\operatorname{deg}_{p}(X)=1$. Hence $\operatorname{deg}_{p}$ is majorized by $\operatorname{deg}$ and is thus finite for each $X \in \mathcal{H}$.

We denote by $H_{n, k}$ the number of linearly inequivalent terms $X$ with weight $\operatorname{deg}(X)=$ $n$ and primitivity $\operatorname{deg}_{p}(X)=k$. Then the generators $H_{k}(x):=\sum_{n \geq k} H_{n, k} x^{n}$ satisfy

$$
H_{j+k}(x) \leq H_{j}(x) H_{k}(x), \quad j, k>0 .
$$

Proof: It is sufficient to show that an element $X \in \mathcal{H}_{j+k}$ may be labelled by those terms in $\Delta(X)$ that are in $\mathcal{H}_{j} \otimes \mathcal{H}_{k}$. To prove this, suppose that $X_{1}$ and $X_{2}$ give the same terms in $\mathcal{H}_{j} \otimes \mathcal{H}_{k}$. Now observe that $U_{j, k}:=U_{j-1} \otimes U_{k-1}$ projects onto $\mathcal{H}_{j} \otimes \mathcal{H}_{k}$, giving $U_{j, k} \circ \Delta\left(X_{1}-X_{2}\right)=0$. Finally, observe that coassociativity gives

$$
0=U_{j, k} \Delta\left(X_{1}-X_{2}\right)=P^{\otimes(j+k)} \circ \Delta^{j+k-1}\left(X_{1}-X_{2}\right):=U_{k+j-1}\left(X_{1}-X_{2}\right)
$$

which shows that $X_{1}-X_{2}$ is $(j+k-1)$-primitive and hence that $X_{1}$ and $X_{2}$ are equivalent elements of $\mathcal{H}_{j+k}$.

In consequence of (22) we obtain

$$
H_{k}(x) \leq\left[H_{1}(x)\right]^{k}
$$

This reflects the fact that the terms in $\Delta^{k-1}(X)$ which belong to $\mathcal{H}_{1}^{\otimes k}$ are sufficient to label elements $X \in \mathcal{H}_{k}$. The remarkable feature of the Hopf algebra of rooted trees, to be proved below, is that all the elements of $\mathcal{H}_{1}^{\otimes k}$ are necessary to label elements of $\mathcal{H}_{k}$.

As a further comment, we note that (24) may be strengthened if the Hopf algebra is cocommutative, since then the order of labels is immaterial. In the case of $\mathcal{H}_{\text {ladder }}$, with $\bar{H}_{1}(x)=x /(1-x)$, one thus obtains

$$
\bar{H}_{k}(x) \leq \prod_{j \leq k} \frac{x}{1-x^{j}}
$$

which is in fact saturated by Table 1 . 


\subsection{Saturation}

We now seek to prove that $H_{k}(x)=\left[H_{1}(x)\right]^{k}$ in the case that $\mathcal{H}=\mathcal{H}_{R}$ is the Hopf algebra of undecorated rooted trees.

First we prove that $\operatorname{deg}_{p}\left(X_{j} X_{k}\right)=\operatorname{deg}_{p}\left(X_{j}\right)+\operatorname{deg}_{p}\left(X_{k}\right)$.

Proof: Suppose that $X_{j} \in \mathcal{H}_{j}$ and $X_{k} \in \mathcal{H}_{k}$. Then

$$
U_{j+k-1}\left(X_{j} X_{k}\right)=P^{\otimes(j+k)}\left(\Delta^{j+k-1}\left(X_{j}\right) \Delta^{j+k-1}\left(X_{k}\right)\right) \neq 0
$$

contains 1-primitives in all its slots, giving $U_{j+k}\left(X_{j} X_{k}\right)=0$, by coassociativity.

It is instructive to see how this works out for the product $Z X$, when $Z$ is 1 -primitive and $X$ is $k$-primitive. Then

$$
\Delta^{k}(Z)=\sum_{j=1}^{k+1} e \otimes \ldots \otimes Z_{\left.\right|_{j-\text { th place }}} \otimes \ldots e
$$

consists of all $k+1$ terms with a single $Z$ and $k$ empty trees. As $X$ is $k$-primitive,

$$
\Delta^{k}(X)=\sum_{j=1}^{k+1} \sum_{i_{j}} X_{i_{j}}^{(1)} \otimes \ldots \otimes e_{\left.\right|_{j-\text { th place }}} \otimes \ldots X_{i_{j}}^{(k+1)}+\ldots
$$

with the final ellipsis denoting omission of terms that contain more than one $e$. The latter make no contribution to

$$
U_{k}(Z X)=\sum_{j=1}^{k+1} \sum_{i_{j}} X_{i_{j}}^{(1)} \otimes \ldots \otimes X_{i_{j}}^{(j-1)} \otimes Z_{\left.\right|_{j-\text { th place }}} \otimes X_{i_{j}}^{(j+1)} \otimes \ldots \otimes X_{i_{j}}^{(k+1)}
$$

where $Z$ replaces a single $e$. By construction (29) has all its entries, namely $Z$ or $X_{i_{j}}^{(r)}$, in $\mathcal{H}_{1}$. Hence $U_{k+1}(Z X)=0$ and $\operatorname{deg}_{p}(Z X)=k+1$.

Iterating this result one immediately concludes that $\operatorname{deg}_{p}\left(X_{1} \ldots X_{k}\right)=k$, for 1primitive elements $X_{1}, \ldots, X_{k}$. This does not, of itself, allow us to conclude that $H_{k}(x)=$ $\left[H_{1}(x)\right]^{k}$, since the products are commutative. Thus there are fewer $k$-fold products of 1 -primitives than there are $k$-primitives.

To appreciate what is needed in the next step, we pause to consider $\mathcal{H}_{\mathrm{CM}}$. Its 1 primitives are $\delta_{1}$ and $\widetilde{\delta}_{2}=\delta_{2}-\frac{1}{2} \delta_{1}^{2}$. From these we can form the 2-primitive products $\delta_{1}^{2}, \delta_{1} \widetilde{\delta}_{2}$, and $\widetilde{\delta}_{2}^{2}$. Table 2 shows that there is a further inequivalent 2-primitive, at weight $n=3$. Direct computation shows that it may be taken as $\widetilde{\delta}_{3}=\delta_{3}-\frac{1}{2} \delta_{1}^{3}$. Then we may form 6 inequivalent 3 -primitive products, namely $\delta_{1}^{3}, \delta_{1}^{2} \widetilde{\delta}_{2}, \delta_{1} \widetilde{\delta}_{3}, \delta_{1} \widetilde{\delta}_{2}^{2}, \widetilde{\delta}_{2} \widetilde{\delta}_{3}$ and $\widetilde{\delta}_{2}^{3}$. Table 2 shows that there is only one more 3 -primitive, at weight $n=4$. It may be taken as $\widetilde{\delta}_{4}=\delta_{4}-\frac{3}{4} \delta_{1}^{4}$. The absence of a further inequivalent 3 -primitive at weight $n=5$ means that $\widetilde{H}_{3}(x)<\widetilde{H}_{1}(x) \widetilde{H}_{2}(x)$. This exercise reveals the filtration of the bigrading of Table 2 : the generator is

$$
\sum_{n, k} \widetilde{H}_{n, k} x^{n} y^{k}=\frac{1}{1-x y} \prod_{k>0} \frac{1}{1-x^{k+1} y^{k}}
$$


corresponding to products of $l_{1}$ and

$$
\widetilde{\delta}_{k+1}=N^{k}\left(l_{1}\right)-\frac{k !}{2^{k}} l_{1}^{k+1}
$$

with $k$-primitivity achieved by a subtraction at weight $n=k+1>1$. At $y=1$, the filtration (30) agrees with the ladder filtration

$$
\sum_{n, k} \bar{H}_{n, k} x^{n} y^{k}=\prod_{k>0} \frac{1}{1-x^{k} y}
$$

generated by products of the 1-primitives (8).

Now consider the highly nontrivial filtration of the bigrading of rooted trees. Let $P_{n, k}$ be the number of weight- $n$ elements of $\mathcal{H}_{k}$ that cannot be expressed as products of elements of $\left\{\mathcal{H}_{j} \mid j<k\right\}$. Then

$$
H(x, y):=\sum_{n, k} H_{n, k} x^{n} y^{k}=\prod_{n, k} \frac{1}{\left(1-x^{n} y^{k}\right)^{P_{n, k}}}
$$

with $P_{n, k}$ telling us how many linearly independent combinations of weight- $n$ trees may be made $k$-primitive, but not $(k-1)$-primitive, by suitable subtractions of products of trees of lesser weight. Setting $y=1$, taking logs, and using the unique property (2) of the enumeration of rooted trees, we obtain

$$
\sum_{n} r_{n} \log \left(1-x^{n}\right)=\log x-\log R(x)=\sum_{n, k} P_{n, k} \log \left(1-x^{n}\right)
$$

and hence $r_{n}=\sum_{k} P_{n, k}$.

Table 4 gives the filtration implied by $H_{k}(x)=\left[H_{1}(x)\right]^{k}$. The column generators are

$$
P_{k}(x):=\sum_{n} P_{n, k} x^{n}=\sum_{j \mid k} \frac{\mu(j)}{k}\left(1-\prod_{n}\left(1-x^{n j}\right)^{r_{n}}\right)^{k / j}
$$

where the Möbius function $\mu(j)$ vanishes if $j$ is divisible by a square and is equal to $(-1)^{p}$ when $j$ is the product of $p$ distinct primes.

To proceed, we use the Hochschild property of $B_{+}$, namely

$$
\Delta \circ B_{+}=B_{+} \otimes e+\left(\mathrm{id} \otimes B_{+}\right) \circ \Delta
$$

which follows from the action of the coproduct (3) on the trees produced by $B_{+}$, using $B_{-} \circ B_{+}=$id. Taking care to note that

$$
C:=B_{+} \circ B_{-} \neq B_{-} \circ B_{+}=\mathrm{id}
$$

we obtain

$$
\Delta \circ B_{-}=\left(\text {id } \otimes B_{-}\right) \circ \Delta \circ C
$$


by composition of (36) with id $\otimes B_{-}$on the left and $B_{-}$on the right. It follows from (36) that if $X$ is $k$-primitive, then $B_{+}(X)$ has primitivity no greater than $k+1$.

Proof: Suppose that $X \in \mathcal{H}_{k}$. Then repeated application of (36) gives

$$
U_{k+1} \circ B_{+}(X)=\left(P^{\otimes(k+1)} \otimes B_{+}\right) \circ \Delta^{k+1}(X)=0
$$

since every term in $\Delta^{k+1}(X)$ contains at least two $e$ 's, of which at most one is promoted to $l_{1}$ by $B_{+}$.

The presence of $C$ in (38) frustrates a parallel attempt to show that $B_{-}$decreases primitivity. Rather, we found that the kernel of $B_{-}$is an object of great interest. The action of $B_{-}$on a nonempty tree $t$ is simple: it removes the root to produce, in general, a forest of rooted trees, each of whose roots was originally connected to the root of $t$ by a single edge. Since $B_{-}$obeys the Leibniz rule

$$
B_{-}\left(X_{1} X_{2}\right)=X_{1} B_{-}\left(X_{2}\right)+X_{2} B_{-}\left(X_{1}\right), \quad B_{-}(e)=0
$$

its action on forests is less trivial. The action of $B_{-}$on a tree, $t$, is undone by $B_{+}$, giving $C(t):=B_{+}\left(B_{-}(t)\right)=t$. On a forest of more than one tree, $C$ does not degenerate to the identity map. It is this that makes the Hopf algebra of rooted trees such an amazingly rich structure. Another important feature is that the kernels of $B_{-}$and $C$ coincide, since $C:=B_{+} \circ B_{-}$and $B_{-}=B_{-} \circ C$. Moreover, $C$ is idempotent, since

$$
(C-\mathrm{id}) \circ C=B_{+} \circ\left(B_{-} \circ B_{+}-\mathrm{id}\right) \circ B_{-}=0 .
$$

Hence there are two special types of object: trees, for which $C$ acts like the identity, and those linear combinations of forests that lie in the kernel of $C$. We shall show that the latter are the key to the filtration $P_{n, k}$ of Table 4 . The first step is to prove that $C(X)=0$ for every $X \in \mathcal{H}_{1}$ with weight $n>1$.

Proof: The coproduct of tree $t$ has the form

$$
\Delta(t)=t \otimes e+B_{-}(t) \otimes l_{1}+\ldots
$$

where the ellipsis denotes terms with weight $n>1$ on the right. Now consider a forest $F=\prod_{j} t_{j}$. The Leibniz rule (40) gives

$$
\Delta(F)=\prod_{j} \Delta\left(t_{j}\right)=F \otimes e+B_{-}(F) \otimes l_{1}+\ldots
$$

and hence $\Delta(X)$ contains $B_{-}(X) \otimes l_{1}$, for all $X \in \mathcal{H}_{R}$. Now suppose that $X \in \mathcal{H}_{1}$ has no weight-1 term. Then $\Delta(X)=X \otimes e+e \otimes X$ requires that $B_{-}(X)=0$ and hence that $X$ is in the kernel of $C$.

To get acquainted with the problem in hand, consider a pair of 1-primitives, $X_{1}$ and $X_{2}$. Their product is 2-primitive, giving

$$
U_{1}\left(X_{1} X_{2}\right)=X_{1} \otimes X_{2}+X_{2} \otimes X_{1}
$$


For every such pair, we require another 2-primitive construct, say $W\left(X_{1}, X_{2}\right)$, giving

$$
U_{1} \circ W\left(X_{1}, X_{2}\right)=X_{1} \otimes X_{2}-X_{2} \otimes X_{1} .
$$

This does not uniquely define $W\left(X_{1}, X_{2}\right)$, since we may add to any solution of (45) any combination of 1-primitives. The operative question is whether a solution exists, for each pair of distinct 1-primitives. This question does not arise in the ladder subalgebra, which is cocommutative. It is easily answered in the Connes-Moscovici subalgebra, where the asymmetry of

$$
U_{1}\left(\widetilde{\delta}_{3}\right)=3 \delta_{1} \otimes \widetilde{\delta}_{2}+\widetilde{\delta}_{2} \otimes \delta_{1}
$$

makes it simple to solve the single case of (45) by

$$
W\left(\delta_{1}, \widetilde{\delta}_{2}\right)=\widetilde{\delta}_{3}-2 \widetilde{\delta}_{2} \delta_{1}=B_{+}\left(l_{1}^{2}\right)+l_{3}-2 l_{2} l_{1}+\frac{1}{2} l_{1}^{3} .
$$

More generally, the $k$-primitive nonproduct term $\widetilde{\delta}_{k+1}$ accounts for the leading diagonal $P_{k+1, k}=1$ of Table 4 .

In the full Hopf algebra, we must show the existence of $P_{n, 2}$ asymmetric pairings enumerated by

$$
P_{2}(x):=\sum_{n} P_{n, 2} x^{n}=\frac{1}{2}\left[H_{1}(x)\right]^{2}-\frac{1}{2} H_{1}\left(x^{2}\right)=x^{3}+x^{4}+3 x^{5}+5 x^{6}+13 x^{7}+28 x^{8}+\ldots
$$

Part of what is required is clearly provided by

$$
W\left(l_{1}, X\right)=l_{1} X-2 B_{+}(X)
$$

since (36) shows that

$$
U_{1} \circ W\left(l_{1}, X\right)=l_{1} \otimes X+X \otimes l_{1}-2\left(P \circ B_{+}\right)(X \otimes e+e \otimes X)=l_{1} \otimes X-X \otimes l_{1}
$$

has the desired antisymmetry. By this means we easily construct the elements of $\mathcal{H}_{2}$ with weight $n<5$ from products of 1-primitives and the action of $B_{+}$on 1-primitives.

At weights $n \geq 5$ we need a further construction. There are $P_{5,2}=3$ weight- 5 nonproduct 2-primitives, but only $H_{4,1}=2$ weight-4 1-primitives on which to act with $B_{+}$. We lack, thus far, a way of constructing $W\left(p_{2}, p_{3}\right)$, where $p_{2}=l_{2}-\frac{1}{2} l_{1}^{2}$ and $p_{3}=l_{3}-l_{2} l_{1}+\frac{1}{3} l_{1}^{3}$ are the 1 -primitives at weights $n=2,3$, common to the cocommutative subalgebra $\mathcal{H}_{\text {ladder }}$. At weight $n=6$, we lack $W\left(p_{2}, p_{4}\right)$ and $W\left(p_{2}, p_{4}^{\prime}\right)$, where

$$
\begin{aligned}
& p_{4}=l_{4}-l_{3} l_{1}-\frac{1}{2} l_{2}^{2}+l_{2} l_{1}^{2}-\frac{1}{4} l_{1}^{4}, \\
& p_{4}^{\prime}=B_{+}\left(2 l_{2} l_{1}-B_{+}\left(l_{1}^{2}\right)-l_{1}^{3}\right)+l_{1} B_{+}\left(l_{1}^{2}\right)-l_{2}^{2},
\end{aligned}
$$

are the weight- 4 ladder and nonladder 1 -primitives enumerated by $H_{4,1}=2$. It is simple to check that they are annihilated by the Leibniz action of $B_{-}$, using $B_{-} \circ B_{+}=$id and $B_{-}\left(l_{n}\right)=l_{n-1}$ with $l_{0}:=e$ evaluating to unity.

At this juncture, it is instructive to compare Tables 3 and 4, which reveal that

$$
\begin{aligned}
& P_{n, 2} \leq 2 H_{n-1,1} \\
& P_{n, k} \leq H_{n-1, k-1}, \quad k>2 .
\end{aligned}
$$


In the Appendix, we show that these inequalities persist at large $n$, thanks to the fact that the Otter constant $c:=\lim _{n \rightarrow \infty} r_{n+1} / r_{n}=2.955765 \ldots$ is slightly less than 3 . Thus it is conceivable that for $k>2$ the action of $B_{+}$might generate $P_{k}(x)$ from $x H_{k-1}(x)$, but it is quite impossible for it to do this job at $k=2$. It appears from (53) that we need a second operator that increases $n$ and $k$ by unity.

\subsection{Natural growth by a single node}

There is a clear candidate for the second operator: the natural growth operator $N$, which appends a single node in all possible ways, and hence obeys a Leibniz rule.

The commutators of $N$ with $B_{ \pm}$are easily found, since we need only consider what is happening at the root. Defining the operator $L$ by $L(X):=l_{1} X$, we obtain

$$
\begin{aligned}
{\left[N, B_{+}\right] } & =B_{+} \circ L, \\
{\left[B_{-}, N\right] } & =L \circ B_{-}, \\
{[N, C] } & =\left[N, B_{+}\right] \circ B_{-}-B_{+} \circ\left[B_{-}, N\right]=0 .
\end{aligned}
$$

The natural growth operator is a wonderful thing: it commutes with $B_{+} \circ B_{-}$, the operator that makes the Hopf algebra so structured; hence it preserves the kernel of $B_{-}$; like $B_{-}$, it acts as a derivative; like $B_{+}$, it adds a node and increases the degree of primitivity; finally, it identifies the unique [2] noncocommutative Hopf subalgebra $\mathcal{H}_{\mathrm{CM}}$, with linear basis $\delta_{n}:=N^{n-1}\left(l_{1}\right)$.

Constructing $N\left(p_{4}\right)$ and $N\left(p_{4}^{\prime}\right)$, we verified that they are in the kernel of $U_{2}$ and the range of $U_{1}$. It might thus appear that some linear combination of them with $B_{+}\left(p_{4}\right), B_{+}\left(p_{4}^{\prime}\right)$ and the product terms $\left\{p_{1} p_{4}, p_{1} p_{4}^{\prime}, p_{2} p_{3}\right\}$ solves the problem of constructing $W\left(p_{2}, p_{3}\right)$. Remarkably, this turns out not to be the case. Rather, we find that application of

$$
S_{1}:=N+\left(B_{+}-L\right) \circ Y
$$

to a 1-primitive gives a 1-primitive of higher weight. Here $Y$ is the grading operator, which multiplies each tree by its weight and operates on products by a Leibniz rule. Thus $N\left(p_{4}\right)$ and $N\left(p_{4}^{\prime}\right)$ are linear combinations of $\left\{B_{+}\left(p_{4}\right), B_{+}\left(p_{4}^{\prime}\right), p_{1} p_{4}, p_{1} p_{4}^{\prime}\right\}$ and 1-primitives. Instead of constructing the missing weight-5 nonproduct 2-primitive, we discovered how to generate all the 1 -primitives with weight $n \leq 5$. We have $L(e)=p_{1}=l_{1}$, at $n=1$; $S_{1}\left(p_{1}\right)=2 p_{2}$, at $n=2 ; S_{1}\left(p_{2}\right)=3 p_{3}$, at $n=3$. At $n=4$, we obtain $S_{1}\left(p_{3}\right)=4 p_{4}-p_{4}^{\prime}$, to which we adjoin $p_{4}$, from the ladder construction (8) of sect. 3 . Then we obtain the 1-primitives at $n=5$ as $p_{5}, S_{1}\left(p_{4}\right)$ and $S_{1}^{2}\left(p_{3}\right)$.

We then found a generalization of (58), which solves the problem of constructing $W\left(p_{2}, p_{3}\right)$. Operating on a weight- $n$ 1-primitive with

$$
S_{k}:=\left(S_{1}-\frac{k-1}{2}\left(B_{+}-L\right)\right) \circ N^{k-1}
$$

we create a $k$-primitive of weight $n+k$. In particular,

$$
W\left(p_{2}, p_{3}\right)=\frac{8 S_{2}\left(p_{3}\right)-7 N \circ S_{1}\left(p_{3}\right)}{12}-p_{2} p_{3}
$$


completes the construction of weight-5 2-primitives. More generally, we found that

$$
\begin{aligned}
W\left(p_{2}, X_{n}\right) & =\frac{2 O_{2}\left(X_{n}\right)}{n(n+1)}-p_{2} X_{n} \\
O_{2} & :=S_{2} \circ(Y+\mathrm{id})-N \circ S_{1} \circ\left(Y+\frac{1}{2} \mathrm{id}\right)
\end{aligned}
$$

gives $U_{1} \circ W\left(p_{2}, X_{n}\right)=p_{2} \otimes X_{n}-X_{n} \otimes p_{2}$, where $X_{n}$ is a 1-primitive with weight $n$. We remark that (61) lies in the kernel of $C$, for all $n>1$.

However, it is not yet clear how to generalize this construction to obtain, for example, $W\left(p_{3}, p_{4}\right)$ and $W\left(p_{3}, p_{4}^{\prime}\right)$ at weight $n=7$. They key to this issue is an extension' of the concept of natural growth.

\subsection{Natural growth by appending sums of forests}

Let $F$ be a forest. We define $N_{F}(X)$ to be the sum of forests obtained by appending $F$ to every node of $X$, in turn. To append $F=\prod_{j} t_{j}$ to a particular node, one connects the roots of all the $t_{j}$ to that node. We note that $N_{F}$ obeys a Leibniz rule, with $N_{F}(e)=0$ and $N_{F}\left(l_{1}\right)=B_{+}(F)$. We have already encountered two examples, namely the grading operator $Y:=N_{e}$, which merely counts nodes, and the simplest natural growth operator $N:=N_{l_{1}}$, which appends a single node. Finally, with $Z=F_{1}+F_{2}$, we make $N_{Z}:=$ $N_{F_{1}}+N_{F_{2}}$ linear in its subscript, as well as its argument.

The commutation relations $(55,56)$ then generalize to

$$
\begin{aligned}
& {\left[N_{Z}, B_{+}\right]=B_{+} \circ L_{Z}} \\
& {\left[B_{-}, N_{Z}\right]=L_{Z} \circ B_{-}}
\end{aligned}
$$

with $L_{Z}(X):=Z X$. Thus $\left[N_{Z}, C\right]=0$ and $N_{Z}$ preserves the kernel of $C$ for all $Z \in \mathcal{H}_{R}$.

The great virtue of this construct is that it gives

$$
U_{1} \circ N_{Z}(X)=Z \otimes Y(X)
$$

when both $Z$ and $X$ are 1-primitive.

Proof: We use the shorthand notation $\Delta(X)=X \otimes e+e \otimes X+X^{\prime} \otimes X^{\prime \prime}$ for any Hopf algebra element $X$, with the final term denoting a sum over tensor products containing no scalars. Let $Z$ be any 1-primitive. Then

$$
U_{1} \circ N_{Z}(X)=N_{Z}\left(X^{\prime}\right) \otimes X^{\prime \prime}+X^{\prime} \otimes N_{Z}\left(X^{\prime \prime}\right)+\left(L_{Z} \otimes Y\right) \circ \Delta(X)
$$

consists of terms in which $X^{\prime}$ or $X^{\prime \prime}$ grow naturally, with a final contribution where $Z$ is itself completely cut from any node to which it was connected by $N_{Z}$, with the grading operator $Y$ acting on the right, to count the number of cuts. The case with $Z=l_{1}$ was proven in [2], by an analysis of admissible cuts. Here, where $Z$ is 1-primitive, we obtain

\footnotetext{
${ }^{1}$ Our extension of natural growth allows a suitable extension of the Lie algebra dual to $\mathcal{H}_{R}$, as was observed by Alain Connes. This will be presented in a sequel to [7].
} 
a result of the same form, since the internal cuts of $Z$ cancel when $U_{1}(Z)=0$. (A more general formula, for arbitrary $Z$, can be given but is not required here.) When $X$ is 1-primitive, with $X^{\prime}=X^{\prime \prime}=0$, we obtain (65) from $L_{Z} \otimes Y$ acting on the second term of $\Delta(X)=X \otimes e+e \otimes X$

The result (65) immediately proves that $H_{2}(x)=\left[H_{1}(x)\right]^{2}$, since it shows that each pairing $N_{X_{1}}\left(X_{2}\right)$ of 1-primitives gives an element of $\mathcal{H}_{2}$ that is inequivalent to any other pairing. Hence (24) is saturated at $k=2$. Now we define the iteration

$$
V_{k+1}\left(X_{1}, \ldots, X_{k}, X_{k+1}\right):=N_{V_{k}\left(X_{1}, \ldots, X_{k}\right)}\left(X_{k+1}\right)
$$

for $k>0$, with $V_{1}:=$ id. Then, for example, $V_{2}\left(X_{1}, X_{2}\right):=N_{X_{1}}\left(X_{2}\right)$ and

$$
V_{3}\left(X_{1}, X_{2}, X_{3}\right):=N_{N_{X_{1}}\left(X_{2}\right)}\left(X_{3}\right) \neq N_{X_{1}}\left(N_{X_{2}}\left(X_{3}\right)\right) \text {. }
$$

We remark that a Hochschild boundary can be defined for maps $V_{k+1}: \mathcal{H}_{R}^{\otimes(k+1)} \rightarrow \mathcal{H}_{R}$. For this, it is sufficient to define terms of the form $V_{k}\left(X_{1}, \ldots, X_{j} X_{j+1}, \ldots, X_{k+1}\right)$, where one argument is a product. Natural growth by forests supplies this. Consequences will be described in future work. For the present, we are content with the following result.

Theorem: The dimensions $H_{n, k}$ of the bigrading of the Hopf algebra of undecorated rooted trees, by weight $n$ and degree of primitivity $k$, are generated by (11).

Proof: Let $X_{1}, X_{2}, \ldots, X_{k}$ be 1-primitives, which need not be distinct. Then

$$
U_{k-1} \circ V_{k}\left(X_{1}, X_{2}, \ldots, X_{k}\right)=X_{1} \otimes Y\left(X_{2}\right) \otimes \ldots \otimes Y\left(X_{k}\right)
$$

by coassociativity and iteration of the argument that led to (65). Thus $H_{k}(x)=\left[H_{1}(x)\right]^{k}$ saturates (24). Then $H(x, y)=1 /\left(1-H_{1}(x) y\right)$ gives $R(x)=x /\left(1-H_{1}(x)\right)$, at $y=1$. Solving for $H_{1}(x)=1-x / R(x)$, we obtain (1).

\subsection{Comments on the main theorem}

Four comments are in order. The first concerns the enumeration of the filtration. This follows from taking logs in (33), which gives

$$
\log H(x, y)=-\log \left(1-H_{1}(x) y\right)=-\sum_{n, k} P_{n, k} \log \left(1-x^{n} y^{k}\right) .
$$

Equating coefficients of $y^{j}$, and setting $x=z^{1 / j}$, we obtain

$$
\left[H_{1}\left(z^{1 / j}\right)\right]^{j}=\sum_{k \mid j} k P_{k}\left(z^{1 / k}\right)
$$

which is a classic problem in Möbius inversion, yielding (35), after use of (2).

Next, we remark on the number, $C_{n, k}$, of weight- $n$ elements of $\mathcal{H}_{k}$ that are in the kernel of $C:=B_{+} \circ B_{-}$. We have explicitly constructed a filtration of the bigrading, for weights $n<7$, in which the only element with $C(X) \neq 0$ is $l_{1}$. The iteration (67) proves 
that there is no obstacle to continuing this process, since the only restriction imposed by $C \circ V_{k+1}\left(X_{1}, X_{2}, \ldots, X_{k+1}\right)=0$ is $X_{k+1} \neq l_{1}$. Thus $\sum_{n} C_{n, k+1} x^{n}=\left[H_{1}(x)\right]^{k}\left(H_{1}(x)-x\right)$ and the generating function

$$
\sum_{n, k} C_{n, k} x^{n} y^{k}=\frac{(1-x y) R(x)}{(1-y) R(x)+x y}
$$

differs from (11) only by a factor of $1-x y$, which removes $l_{1}$ from the filtration (33). In total, we have $C_{n}:=\sum_{k} C_{n, k}=r_{n+1}-r_{n}$ weight- $n$ solutions to $C(X)=0$. It is easy to see how that comes about: there are $r_{n+1}$ possible forests in $X$, subject to the $r_{n}$ conditions that the coefficient of every tree in $C(X)$ vanishes. The result $C_{n}=r_{n+1}-r_{n}$ proves the independence of these conditions. Hence an element $X$ of the kernel of $C$ is uniquely identified by the contribution $\bar{X}$ that contains no pure trees, since $X=\bar{X}-C(\bar{X})$. Finally, the filtration of the bigrading of the kernel of $C$ differs from that of the full Hopf algebra only by the absence of $l_{1}$. These distinctive features frustrate every attempt to decrease primitivity by the action of $B_{-}$on any nonproduct element except the singlenode tree. One may climb up the ladder of primitivity with great ease, yet descent is impossible, save in one trivial case. In a sense, the second grading is characterized by the profound difficulty of constructing its 1-primitives. At first meeting, this makes it difficult to fathom. Then one realizes that the structure is beautifully tuned to prevent casual construction.

Our third comment concerns the remarkable operator $\mathrm{O}_{2}$ in (62), which provides a way of solving $U_{1} \circ W\left(p_{2}, X\right)=p_{2} \otimes X-X \otimes p_{2}$. A second way is provided by $N_{p_{2}}$. These solutions need not be the same; they may differ by a 1-primitive. In general, they will differ, since $N_{p_{2}}$ acts by a Leibniz rule, while $\mathrm{O}_{2}$ does not. Hence

$$
T_{2}:=O_{2}-N_{p_{2}} \circ(Y+\mathrm{id})
$$

provides a second shift operator that creates 1-primitives, when applied to 1-primitives. It gives information that is not provided by $S_{1}$ in (58). For example, at weight $n=6$ we already know how to construct 4 of the $H_{6,1}=8$ primitives, by applying powers of $S_{1}$ to the ladder primitives constructed in (8). Of the missing 4 , the constructs $T_{2}\left(p_{4}\right)$ and $T_{2} \circ S_{1}\left(p_{3}\right)$ provide 2 . For the remaining 2 , which are now proven to exist, we laboriously solved $U_{1}(X)=0$ at weight $n=6$, working with tensor products of the 38 forests with up to 6 nodes. At first sight, one might hope to add a few more shift operators, to arrive at a set that is sufficient to construct 1-primitives up to some large weight, without having to solve the fearsome explosion of linear equations required by the vanishing of all tensor products in $U_{1}(X)=0$. This seems not to be the case; the construction of 1-primitives appears to be a deeply nontrivial challenge. Asymptotically, no more than a fraction $1 / c$ of what is necessary may be provided by $S_{1}$, and no more than $1 / c^{2}$ by $T_{2}$, which increases weight by 2 units. The number of similarly constructed operators that change weight by $n$ cannot exceed the number $H_{n, 1}$ of weight- $n$ 1-primitives. Constructing a finite number of these, we obtain merely an asymptotic fraction $f<H_{1}(1 / c)=1-1 / c<1$ of what is needed. Hence we envisage no easy route to the construction of 1-primitives, short of solving the tensorial defining property $\Delta(X)=X \otimes e+e \otimes X$. Thereafter, the problem of constructing $k$-primitives is completely solved by (67), which shows that the 
1-primitives of weight $n>1$ are enumerated by those elements of the kernel of $C$ that cannot be generated by any process of natural growth acting on 1-primitives of lesser weight. This negative criterion appears even harder to implement than the tensorial definition $U_{1}(X)=0$, which we were able to solve at $n=9$, by explicit computation of the 98 -dimensional kernel of a $3214 \times 719$ matrix of integers.

Finally, we remark that we have explicit constructions of the bigradings (30,32) of the Connes-Moscovici and ladder subalgebras. In the case of $\mathcal{H}_{\mathrm{CM}}$ we have merely a pair of 1-primitives: $\delta_{1}=l_{1}$ and $\widetilde{\delta}_{2}=N_{\delta_{1}}\left(\delta_{1}\right)-\frac{1}{2} \delta_{1}^{2}$. The only form of natural growth that we are allowed is by a single node: this is the defining restriction. Then we easily construct $\widetilde{\delta}_{k+1}=N_{\delta_{1}}^{k}\left(\delta_{1}\right)-2^{-k} k ! \delta_{1}^{k+1}$ as a nonproduct $k$-primitive of weight $k+1$. This completes the filtration, since any further term would make the number of weight- $n$ products of filtered elements greater than the number of weight- $n$ products of the linear basis. Hence the construction of the Connes-Moscovici bigrading is particularly simple. In the case of $\mathcal{H}_{\text {ladder }}$ the cocommutativity of the ladder restriction (6) of the coproduct means that all $k$-primitives are products at $k>1$. Here the problem of construction is more demanding, since it not clear how to generate an infinite set of 1-primitives. Hence one sees that detailed study of ladder diagrams, most notably by Bob Delbourgo and colleagues [12, 13, 14], addresses a problem more severe than that posed by the ConnesMoscovici prolegomenon to noncommutative geometry: ladder diagrams are a nontrivial infinite subset of perturbative quantum field theory; even after subtractions of products they provide an infinite subset of 1-primitives, when their bigrading is analyzed. Fortunately, our recent work in [8] provides the explicit construction (\&) of the ladder filtration. The reader may try to imagine what might be involved in giving an explicit construction of the 1-primitives of the full Hopf algebra of undecorated rooted trees. Then s/he should contemplate the true challenge of quantum field theory, by recalling that - in physical reality - every node of every rooted tree may be decorated in an infinite number of ways. After half a century, few physicists or mathematicians have even begun to grapple with the true legacy of Dyson, Feynman, Schwinger and Tomonaga.

\section{Prospects}

In this paper, we were content to study the bigrading of the Hopf algebra of undecorated 《4, 8 rooted trees, by the number of nodes and a degree of primitivity analyzed by iterations of the coproduct. The extension of this bigrading to the decorated [3, 7] case is the obvious next step, in our plan to decode the rich structure of mature quantum field theory. The present work makes it clear that the key feature will be the nontriviality of $C:=B_{+} \circ B_{-} \neq B_{-} \circ B_{+}=\mathrm{id}$. In the undecorated case, we have shown that the bifiltration of the Hopf algebra is obtained by adjoining the single-node tree to the bifiltration of the kernel of $C$. The proof of this lies in the powerful generalization (67) of the concept of natural growth, which diagonalizes (69). First results for the commutator $\left[B_{+}, B_{-}\right]=C-$ id of the decorated Hopf algebra of full quantum field theory were recently given in [9]. These increase our hopes that it will not take another 50 years to complete the characterization of the intricate interrelation of combinatorics and analysis that makes 
quantum field theory possible. We firmly believe that further elucidation of its structure has much to offer for wide areas of both physics and mathematics.

Acknowledgements: This study began during the workshop Number Theory and Physics at the ESI in November 1999, where we enjoyed discussions with Pierre Cartier, Werner Nahm, Ivan Todorov and Jean-Bernard Zuber. Work with Alain Connes at the IHES supports the present paper. System management by Chris Wigglesworth enabled accumulation of crucial data, which Neil Sloane's superseeker helped us to decode.

\section{Appendix: asymptotic enumerations}

Here we consider inequalities inferred from Tables 3 and 4 and show that they persist at large weights, thanks to the upper bound $c<3$ on the Otter constant [17.

Asymptotically, the number of rooted trees is given by

$$
r_{n}=c^{n} n^{-3 / 2}(b+O(1 / n))
$$

with Otter constants that we evaluated in 4 :

$$
\begin{aligned}
& b= 0.43992401257102530404090339143454476479808540794011 \\
& 98576534935450226354004204764605379862197779782334 \ldots \\
& c=2.95576528565199497471481752412319458837549230466359 \\
& 65953504724789059647331395749510866682836765813525 \ldots
\end{aligned}
$$

The asymptotic fraction of trees assigned to primitivity $k$ in the filtration of Table 4 is

$$
f_{k}:=\lim _{n \rightarrow \infty} \frac{P_{n, k}}{r_{n}}=\left(1-\frac{1}{c}\right)^{k-1} \frac{1}{c}
$$

while the asymptotic fraction of forests in Table 3 is

$$
g_{k}:=\lim _{n \rightarrow \infty} \frac{H_{n, k}}{r_{n+1}}=\frac{k f_{k}}{c}=\left(1-\frac{1}{c}\right)^{k-1} \frac{k}{c^{2}} .
$$

These follow by using [4] $|1-R(x)|^{2}=O(1-c x)$, near $x=1 / c$. Numerically,

$$
\begin{aligned}
& g_{1}=0.1144616788557279695 \ldots \\
& g_{2}=0.1514735822429146084 \ldots \\
& g_{3}=0.1503401379409753267 \ldots \\
& g_{4}=0.1326357110750687024 \ldots \\
& g_{5}=0.1097026887662558145 \ldots \\
& g_{6}=0.0871054456752243543 \ldots \\
& g_{7}=0.0672417311397409555 \ldots \\
& g_{8}=0.0508484386279160206 \ldots \\
& g_{9}=0.0378509630072558308 \ldots
\end{aligned}
$$


with $k=2$ giving the largest fraction of forests at large $n$. This was not apparent until $n=28$, where we found that $H_{28,2}=20716895918$ exceeds $H_{28,3}=20710700277$.

The asymptotic results establish inequalities $(53,54)$ at large $n$, where it is sufficient that $c<3$. Amusingly, this upper bound and the condition $R(1 / c)=1$ produce a rather tight lower bound

$$
c=\exp \left(\sum_{k>0} \frac{R\left(c^{-k}\right)}{k}\right)>\exp \left(1+\sum_{k>1} \frac{1}{\left(3^{k}-1\right) k}\right)>2.943
$$

from the rather loose lower bound $R(x) \geq R_{\text {ladder }}(x)=x /(1-x)$.

\section{References}

[1] D. Kreimer, Adv. Theor. Math. Phys. 2 (1998) 303, q-alg/9707029.

[2] A. Connes, D. Kreimer, Comm. Math. Phys. 199 (1998) 203, hep-th/9808042.

[3] D. Kreimer, Comm. Math. Phys. 204 (1999) 669, hep-th/9810022.

[4] D.J. Broadhurst, D. Kreimer, J. Symb. Comput. 27 (1999) 581, hep-th/9810087.

[5] D. Kreimer, Adv. Theor. Math. Phys. 3.3 (1999), hep-th/9901099.

[6] A. Connes, D. Kreimer, JHEP 9909 (1999) 024, hep-th/9909126.

[7] A. Connes, D. Kreimer, Comm. Math. Phys., in press, hep-th/9912092.

[8] D.J. Broadhurst, D. Kreimer, Phys. Lett. B, in press, hep-th/9912093.

[9] D. Kreimer, hep-th/9912290.

[10] A. Connes, H. Moscovici, Hopf Algebras, cyclic Cohomology and the transverse Index Theorem, IHES/M/98/37, math.DG/9806109.

[11] D. Kreimer, J. Knot Th. Ram. 6 (1997) 479, q-alg/9607022.

[12] R. Delbourgo, A. Kalloniatis, G. Thompson, Phys. Rev. D54 (1996) 5373, hep-th/9605107.

[13] R. Delbourgo, D. Elliott, D.S. McAnally, Phys. Rev. D55 (1997) 5230, hep-th/9611150.

[14] D. Kreimer, R. Delbourgo, Phys. Rev. D60 (1999) 105025, hep-th/9903249.

[15] N.J.A. Sloane, On-line Encyclopedia of Integer Sequences, http://www.research.att.com/njas/sequences

[16] A.C. Hearn, REDUCE User's Manual, Version 3.7, March 1999.

[17] R. Otter, Annals Math. 49 (1948) 583. 
Table 1: Dimensions $\bar{H}_{n, k}$ of the bigrading the cocommutative subalgebra, $\mathcal{H}_{\text {ladder }}$

\begin{tabular}{r|rrrrrrrrrrrrrrrrrrr} 
& 1 & 2 & 3 & 4 & 5 & 6 & 7 & 8 & 9 & 10 & 11 & 12 & 13 & 14 & 15 & 16 & 17 & 18 & 19 \\
\hline 1 & 1 & & & & & & & & & & & & & & & & & & \\
2 & 1 & 1 & & & & & & & & & & & & & & & & \\
3 & 1 & 1 & 1 & & & & & & & & & & & & & & & \\
4 & 1 & 2 & 1 & 1 & & & & & & & & & & & & & & \\
5 & 1 & 2 & 2 & 1 & 1 & & & & & & & & & & & & & \\
6 & 1 & 3 & 3 & 2 & 1 & 1 & & & & & & & & & & & & \\
7 & 1 & 3 & 4 & 3 & 2 & 1 & 1 & & & & & & & & & & & \\
8 & 1 & 4 & 5 & 5 & 3 & 2 & 1 & 1 & & & & & & & & & & \\
9 & 1 & 4 & 7 & 6 & 5 & 3 & 2 & 1 & 1 & & & & & & & & & \\
10 & 1 & 5 & 8 & 9 & 7 & 5 & 3 & 2 & 1 & 1 & & & & & & & & \\
11 & 1 & 5 & 10 & 11 & 10 & 7 & 5 & 3 & 2 & 1 & 1 & & & & & & \\
12 & 1 & 6 & 12 & 15 & 13 & 11 & 7 & 5 & 3 & 2 & 1 & 1 & & & & & \\
13 & 1 & 6 & 14 & 18 & 18 & 14 & 11 & 7 & 5 & 3 & 2 & 1 & 1 & & & & & \\
14 & 1 & 7 & 16 & 23 & 23 & 20 & 15 & 11 & 7 & 5 & 3 & 2 & 1 & 1 & & & & \\
15 & 1 & 7 & 19 & 27 & 30 & 26 & 21 & 15 & 11 & 7 & 5 & 3 & 2 & 1 & 1 & & & \\
16 & 1 & 8 & 21 & 34 & 37 & 35 & 28 & 22 & 15 & 11 & 7 & 5 & 3 & 2 & 1 & 1 & & \\
17 & 1 & 8 & 24 & 39 & 47 & 44 & 38 & 29 & 22 & 15 & 11 & 7 & 5 & 3 & 2 & 1 & 1 & & \\
18 & 1 & 9 & 27 & 47 & 57 & 58 & 49 & 40 & 30 & 22 & 15 & 11 & 7 & 5 & 3 & 2 & 1 & 1 & \\
19 & 1 & 9 & 30 & 54 & 70 & 71 & 65 & 52 & 41 & 30 & 22 & 15 & 11 & 7 & 5 & 3 & 2 & 1 & 1
\end{tabular}

Table 2: Dimensions $\widetilde{H}_{n, k}$ of the bigrading the noncocommutative subalgebra, $\mathcal{H}_{\mathrm{CM}}$

\begin{tabular}{r|rrrrrrrrrrrrrrrrrrr} 
& 1 & 2 & 3 & 4 & 5 & 6 & 7 & 8 & 9 & 10 & 11 & 12 & 13 & 14 & 15 & 16 & 17 & 18 & 19 \\
\hline 1 & 1 & & & & & & & & & & & & & & & & & \\
2 & 1 & 1 & & & & & & & & & & & & & & & \\
3 & & 2 & 1 & & & & & & & & & & & & & & & \\
4 & & 1 & 3 & 1 & & & & & & & & & & & & & & \\
5 & & 2 & 4 & 1 & & & & & & & & & & & & & \\
6 & & & 1 & 4 & 5 & 1 & & & & & & & & & & & & \\
7 & & & & 2 & 6 & 6 & 1 & & & & & & & & & & & & \\
8 & & & & 1 & 4 & 9 & 7 & 1 & & & & & & & & & & \\
9 & & & & 2 & 7 & 12 & 8 & 1 & & & & & & & & & \\
10 & & & & 1 & 4 & 11 & 16 & 9 & 1 & & & & & & & & \\
11 & & & & & 2 & 7 & 16 & 20 & 10 & 1 & & & & & & & \\
12 & & & & & 1 & 4 & 12 & 23 & 25 & 11 & 1 & & & & & & \\
13 & & & & & & 2 & 7 & 18 & 31 & 30 & 12 & 1 & & & & & \\
14 & & & & & & 1 & 4 & 12 & 27 & 41 & 36 & 13 & 1 & & & & \\
15 & & & & & & & 2 & 7 & 19 & 38 & 53 & 42 & 14 & 1 & & & \\
16 & & & & & & & 1 & 4 & 12 & 29 & 53 & 67 & 49 & 15 & 1 & & \\
17 & & & & & & & & 2 & 7 & 19 & 42 & 71 & 83 & 56 & 16 & 1 & & \\
18 & & & & & & & & 1 & 4 & 12 & 30 & 60 & 94 & 102 & 64 & 17 & 1 & \\
19 & & & & & & & & & 2 & 7 & 19 & 44 & 83 & 121 & 123 & 72 & 18 & 1
\end{tabular}


Table 3: Dimensions $H_{n, k}$ of the bigrading of the Hopf algebra of rooted trees, $\mathcal{H}_{R}$

\begin{tabular}{l|rrrrrrrrrrrrr} 
& 1 & 2 & 3 & 4 & 5 & 6 & 7 & 8 & 9 & 10 & 11 & 12 & 13 \\
\hline 1 & 1 & & & & & & & & & & & \\
2 & 1 & 1 & & & & & & & & & & \\
3 & 1 & 2 & 1 & & & & & & & & & \\
4 & 2 & 3 & 3 & 1 & & & & & & & & \\
5 & 3 & 6 & 6 & 4 & 1 & & & & & & & \\
6 & 8 & 11 & 13 & 10 & 5 & 1 & & & & & & & \\
7 & 16 & 26 & 27 & 24 & 15 & 6 & 1 & & & & & \\
8 & 41 & 58 & 63 & 55 & 40 & 21 & 7 & 1 & & & & \\
9 & 98 & 142 & 148 & 132 & 100 & 62 & 28 & 8 & 1 & & & \\
10 & 250 & 351 & 363 & 322 & 251 & 168 & 91 & 36 & 9 & 1 & & & \\
11 & 631 & 890 & 912 & 804 & 635 & 444 & 266 & 128 & 45 & 10 & 1 & & \\
12 & 1646 & 2282 & 2330 & 2051 & 1625 & 1167 & 742 & 402 & 174 & 55 & 11 & 1 & \\
13 & 4285 & 5948 & 6036 & 5304 & 4220 & 3072 & 2030 & 1184 & 585 & 230 & 66 & 12 & 1
\end{tabular}

Table 4: Filtration $P_{n, k}$ of the bigrading of $\mathcal{H}_{R}$

\begin{tabular}{l|rrrrrrrrrrrr} 
& 1 & 2 & 3 & 4 & 5 & 6 & 7 & 8 & 9 & 10 & 11 & 12 \\
\hline 1 & 1 & & & & & & & & & & \\
2 & 1 & & & & & & & & & & \\
3 & 1 & 1 & & & & & & & & & \\
4 & 2 & 1 & 1 & & & & & & & & \\
5 & 3 & 3 & 2 & 1 & & & & & & & \\
6 & 8 & 5 & 4 & 2 & 1 & & & & & & & \\
7 & 16 & 13 & 9 & 6 & 3 & 1 & & & & & \\
8 & 41 & 28 & 21 & 13 & 8 & 3 & 1 & & & & \\
9 & 98 & 71 & 49 & 33 & 20 & 10 & 4 & 1 & & & \\
10 & 250 & 174 & 121 & 79 & 50 & 27 & 13 & 4 & 1 & & \\
11 & 631 & 445 & 304 & 201 & 127 & 74 & 38 & 16 & 5 & 1 & & \\
12 & 1646 & 1137 & 776 & 510 & 325 & 192 & 106 & 49 & 19 & 5 & 1 \\
13 & 4285 & 2974 & 2012 & 1326 & 844 & 512 & 290 & 148 & 65 & 23 & 6 & 1
\end{tabular}

